\title{
L'édition numérique de la Chronique de Giovanni della Grossa (milieu Xve siècle). Un projet de recherche pluridisciplinaire sur la Corse médiévale
}

Vannina Marchi van Cauwelaert, Jean-André Cancellieri, Lucie Arrighi, Marc Bonnant, Émilie Tomas et Michèle Ferrara

\section{(2) OpenEdition}

\section{Édition électronique}

URL : https://journals.openedition.org/cem/17847

DOI : $10.4000 / \mathrm{cem} .17847$

ISSN : 1954-3093

Éditeur

Centre d'études médiévales Saint-Germain d'Auxerre

Référence électronique

Vannina Marchi van Cauwelaert, Jean-André Cancellieri, Lucie Arrighi, Marc Bonnant, Émilie Tomas et Michèle Ferrara, «L'édition numérique de la Chronique de Giovanni della Grossa (milieu xve siècle). Un projet de recherche pluridisciplinaire sur la Corse médiévale », Bulletin du centre d'études médiévales d'Auxerre | BUCEMA [En ligne], 24.2 | 2020, mis en ligne le 19 décembre 2020, consulté le 22 septembre 2022. URL : http://journals.openedition.org/cem/17847 ; DOI : https://doi.org/10.4000/cem. 17847

Ce document a été généré automatiquement le 22 septembre 2022.

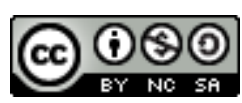

Creative Commons - Attribution - Pas d'Utilisation Commerciale - Partage dans les Mêmes Conditions 4.0 International - CC BY-NC-SA 4.0

https://creativecommons.org/licenses/by-nc-sa/4.0/ 


\section{L'édition numérique de la Chronique de Giovanni della Grossa (milieu $\mathrm{XV}^{\mathrm{e}}$ siècle). Un projet de recherche pluridisciplinaire sur la Corse médiévale}

Vannina Marchi van Cauwelaert, Jean-André Cancellieri, Lucie Arrighi, Marc Bonnant, Émilie Tomas et Michèle Ferrara

\section{NOTE DE L'AUTEUR}

Intitulé Les espaces de la Corse médiévale [ECM], ce projet de recherche, financé par la Collectivité de Corse dans le cadre du programme FEDER (2018-2021) est porté par l'UMR 6240 LISA. Coordonné par Vannina Marchi van Cauwelaert et Jean-André Cancellieri, il réunit : quatre historiens (J.-A. Cancellieri, V. Marchi, M. Ferrara et C. Zedda) ; une philologue (L. Arrighi, EA 4083 CLEA) ; une archéologue (É. Tomas, Arkemine) ; un informaticien - M. Bonnant, Cyrnoweb - ; trois doctorants et quatre étudiants de master. Il bénéficie également de la collaboration du Service régional de l'archéologie (L. Sévègnes), de l'UMS ARCHÉOvISION (L. Espinasse), de l'Opera del Vocabolario Italiano (Pär Larson, CNR), de l'Istituto di Storia dell'Europea Mediterranea (Luciano Gallinari, CNR) et du Laboratorio di Cultura Digitale (E. Salvatori, université de Pise). 
1 En dépit d'un fort développement des recherches universitaires depuis une vingtaine d'années ${ }^{1}$, la Corse médiévale demeure largement méconnue tant du public que des spécialistes. Cette méconnaissance tient principalement à l'extrême rareté des archives locales ${ }^{2}$ qui entrave toute tentative de synthèse malgré la multiplication des sondages

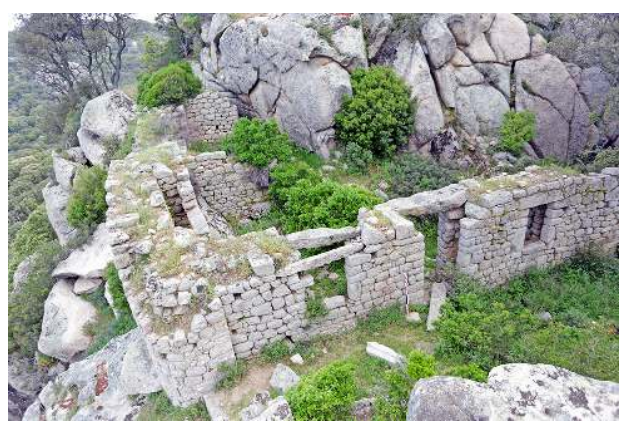
dans les principaux fonds méditerranéens, notamment à Pise $^{3}$, à Gênes ${ }^{4}$ et à Barcelone ${ }^{5}$. Or, si l'archéologie médiévale s'est développée dans l'île, les enquêtes de terrains et les fouilles y demeurent trop rares pour permettre de pallier véritablement les lacunes de la documentation écrite. Dans ce contexte, l'existence d'une Histoire de Corse, écrite au cœur du $\mathrm{Xv}^{\mathrm{e}}$ siècle par un notaire issu d'un village du sud de l'île ${ }^{6}$, constitue un véritable gisement patrimonial. Relatant l'histoire de la Corse depuis ses origines mythiques jusqu'aux années 1460, le texte de Giovanni della Grossa [1388-1464] a longtemps fait le miel des historiens insulaires, avant d'être en partie rejeté par l'historiographie positiviste. Mêlant le mythe à l'histoire, le récit du chroniqueur a peu à peu été écarté au profit des seules documentations archivistique et archéologique, jugées plus dignes de foi. Le recours au texte de Giovanni della Grossa offre néanmoins de nombreux avantages qui en font la source la plus riche pour aborder la Corse médiévale sous l'angle d'une histoire culturelle. En grande partie « histoire des vaincus", le récit de Giovanni della Grossa livre une vision interne sur le Moyen Âge corse dont l'une des ambitions est d'affirmer la légitimité historique des seigneurs insulaires au moment même de leur défaite face à l'Office génois de Saint-Georges ${ }^{7}$. Sa lecture complète avec profit la documentation archivistique et archéologique, en éclairant notamment les systèmes de représentation, les paysages vécus et les réseaux d'alliances. Dans un contexte italien dominé par les chroniques urbaines $^{8}$, ce texte constitue en outre l'un des rares exemples de chronique rurale ayant une portée générale puisqu'il s'agit d'un regard insulaire sur l'histoire politique de l'espace tyrrhénien: la lutte entre chrétienté et islam ( $\mathrm{IX}^{\mathrm{e}}-\mathrm{XI}^{\mathrm{e}}$ siècle) ; l'affirmation de la rivalité entre Pise et Gênes (XII ${ }^{\mathrm{e}}-\mathrm{XIII}^{\mathrm{e}}$ siècles) ; la guerre entre Gênes et l'Aragon (XIV ${ }^{\mathrm{e}}$-milieu XV $\mathrm{XV}^{\mathrm{e}}$ siècle).

2 Le choix d'une édition numérique vise ainsi à confronter les différentes sources chroniques, archives, vestiges archéologiques - afin de faire du récit du chroniqueur l'épine dorsale d'une véritable encyclopédie interactive, et sans cesse actualisée, de la Corse médiévale 9 . En outre, le support numérique facilitera la comparaison des différents manuscrits et de leurs variantes pour une meilleure compréhension du discours politique de Giovanni della Grossa et de ses successeurs ${ }^{10}$.

\section{Le choix du manuscrit et l'établissement du texte}

Si la réputation professionnelle du notaire Giovanni della Grossa est attestée par la documentation génoise $\mathrm{du} \mathrm{Xv}^{\mathrm{e}}$ siècle ${ }^{11}$, celle d'historien de l'île continue d'interroger les spécialistes de la Corse médiévale. L'œuvre historique dans laquelle Giovanni della Grossa est mentionné laisse, en effet, la communauté scientifique perplexe et prudente quant à la pleine attribution du tout premier récit historique corse à ce dernier ${ }^{12}$; les 
seuls manuscrits qui nous sont parvenus datent environ de la fin du XvII jusqu'au milieu du XVIII ${ }^{e}$ siècle. L'examen des manuscrits suggère en outre des remaniements depuis le $\mathrm{xvI}^{\mathrm{e}}$ siècle : le texte se présente sous la forme d'une compilation historique, parsemée d'interpolations et d'apostilles modernes.

4 En 1910, Lucien Letteron fut le premier à faire redécouvrir la « version longue » du récit historique sur le Moyen Âge corse ${ }^{13}$, par rapport à la « version courte » de l'Historia di Corsica imprimée en 1594. À cette époque, il s'agit de quatre manuscrits : 1) le manuscrit Pozzo di Borgo, qui a été conservé par la famille du même nom ; 2) le manuscrit de la famille Galeazzini ${ }^{14}$; 3) le manuscrit italien 839 de la Bibliothèque nationale de France [BnF], copié pour Antonio Buttafoco en 1754 ; 4) le manuscrit $Y$ de la Bibliothèque patrimoniale de Bastia. Se fondant sur l'examen de la graphie qu'il jugeait plus ancienne, Lucien Letteron établit alors le texte à partir du manuscrit Y. En 1999, Antoine Casanova et Mathée Giacomo Marcellesi éditèrent à nouveau le texte assorti d'une première traduction en français ${ }^{15}$.

5 La découverte, dans les années 2010, d'un autre manuscrit chez le libraire Jacques Benelli a permis à Antoine-Marie Graziani de proposer en 2016 une nouvelle édition du texte, accompagnée de sa traduction ${ }^{16}$. L'historien moderniste considère que cette copie est la plus ancienne mise au jour et, partant, la plus conforme à ce qu'aurait pu être le texte original du notaire de la Grossa ${ }^{17}$. C'est sur ce nouveau manuscrit, qui constitue probablement le début de la chaîne des copies de la "version longue » de l'Histoire de Corse, que nous souhaitons fonder cette quatrième édition, dont la version numérique pourrait permettre de mieux distinguer la part médiévale de la part moderne du texte $^{18}$.

6 Contrairement aux éditions précédentes, notre projet entend fonder le choix du manuscrit, en l'occurrence le Benelli, dans le but de retracer la tradition manuscrite de la «version longue" de l'Histoire de Corse ${ }^{19}$. Notre choix repose sur une analyse minutieuse des différentes copies dont le manuscrit Benelli serait la source commune, à l'exception du Galeazzini qui semble avoir été copié à partir du manuscrit de la $\mathrm{BnF}^{20}$. La comparaison des manuscrits Benelli et $Y$ révèle, en effet, des erreurs similaires dues à un problème de lecture. Or, le copiste $\mathrm{Y}$ montre une écriture soignée à la différence de celui du Benelli. Ainsi, lorsque ce dernier distingue difficilement le terme "mimoria ", à cause des quatre jambages [im], il écrit avec hésitation « minioria » que nous retrouvons alors très clairement transcrit de cette manière dans le manuscrit $Y$. Aussi, ce mot ne signifiant rien, le copiste du manuscrit de la BnF corrige-t-il l'erreur en modernisant de surcroît la graphie («memoria »). Cependant, si le manuscrit de la BnF nous aide à faire des choix au moment de la transcription, nous l'avons écarté des deux principaux manuscrits - les manuscrits Benelli et $\mathrm{Y}$ : la graphie du texte est entièrement modernisée, la syntaxe retravaillée, certains passages jugés fautifs sont corrigés, tandis que d'autres, considérés comme énigmatiques, sont résolus par des ajouts ${ }^{21}$. Ces modifications ont été effectuées par le copiste d'Antonio Buttafoco dans le but de donner une meilleure cohérence au texte, mais aussi un sens nouveau, plus conforme aux réalités $\mathrm{du} \mathrm{xvIII}^{\mathrm{e}}$ siècle. Elles laissent formuler l'hypothèse selon laquelle ce manuscrit de 1754 constituerait la phase la plus aboutie d'un projet d'édition envisagé au XVIII siècle. Abandonné pendant les révolutions, ce projet fut envisagé une deuxième fois par Gio'Carlo Gregori (1797-1852) avant d'être repris par Lucien Letteron (fig. 1 et 2). 
Fig. 1 - Schéma des quatre manuscrits de la « version longue » de l'Histoire de Corse.

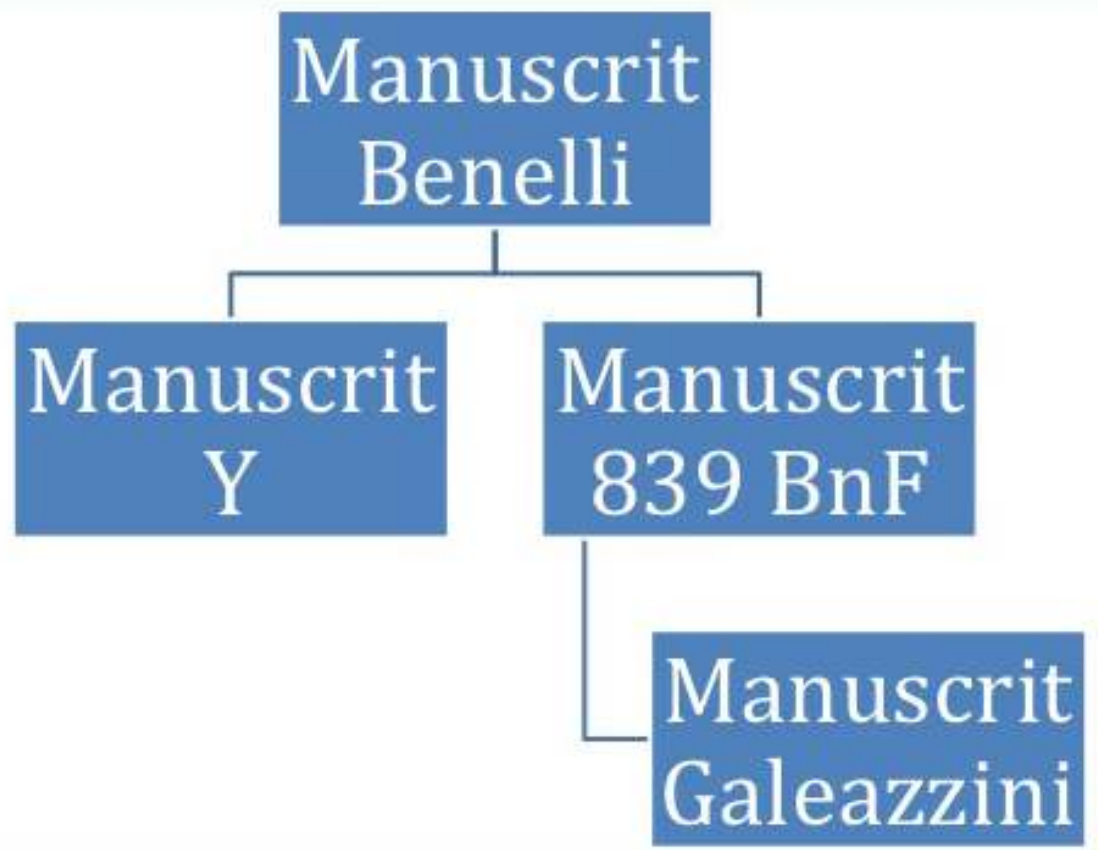

Fig. 2 - À gauche, manuscrit Benelli (cl. Librairie Jacques Benelli); à droite, manuscrit Y (cl. Bibliothèque municipale de Bastia).
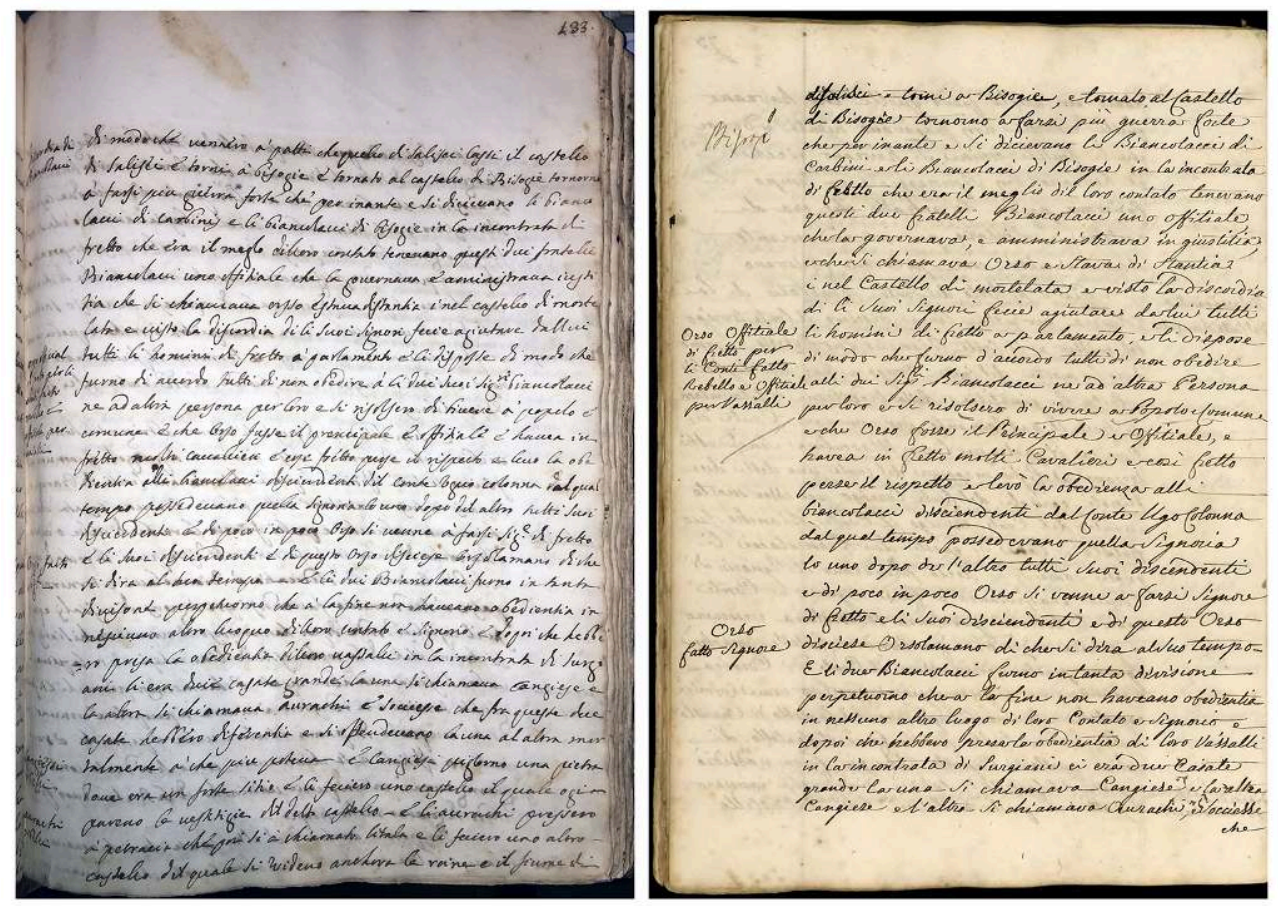


\section{Traitement, numérisation et restitution des données}

7 Dans le but de faciliter la manipulation de la base, c'est le langage de balisage XML qui a été retenu pour le codage du corpus, conformément aux recommandations de la TEI ${ }^{22}$. L'élaboration d'un prototype web a montré qu'un échantillon représentatif de la Chronique $^{23}$, fondé sur la numérisation d'une vingtaine de feuillets du manuscrit de référence contenait en substance tout ce que le langage XML était capable de désigner et de catégoriser dans la perspective d'un traitement numérique. Dans un premier temps, l'extraction des éléments onomastiques a permis de constituer deux index intégrant la Chronique dans le contexte de la Corse médiévale: les anthroponymes, d'une part, ont livré la matière d'une prosopographie générale grâce à la mise en relief des alliances ou des liens de sang; les toponymes, d'autre part, ont exhumé une géographie disparue où d'anciens habitats fortifiés reprennent corps sous le regard de l'archéologie. Ces deux index cardinaux constituent le point d'entrée vers un vaste faisceau de liens hypertextes qui sera continûment augmenté à mesure que l'appareil critique s'enrichira. Les annotations occupant leur propre table dans la base de données pourront, elles aussi, renvoyer à d'autres référents, qu'ils soient internes ou non à la base $^{24}$.

8 L'interface graphique adopte un montage en triptyque : elle est composée de deux blocs latéraux identiques contenant les différents modules accessibles par onglets, et d'un pavé central destiné à afficher de l'information connexe (fiches, médias, etc.) ${ }^{25}$. Ce dernier est exhaussé d'un moteur de recherche à auto-complétion, pour l'heure limité aux seules données onomastiques, mais qui, à terme, couvrira l'ensemble des tables de la base avec options de recherche par critères. La simplicité ergonomique de l'interface entend répondre aux prescriptions d'accessibilité en vigueur ${ }^{26}$, au-delà des contraintes qu'un support traditionnel imposerait du fait de sa linéarité. Plusieurs modules font appel à une variante open source de la technologie Deep Zoom $^{27}$ pour permettre une consultation des contenus en mode image dans leur pleine profondeur. C'est le cas, par exemple, pour les fac-similés des manuscrits - scans en haute définition - et pour la cartographie - Plan Terrier ${ }^{28}$ géo-référencé ${ }^{29}$. Ce prototype a vocation à préfigurer les aspects fonctionnels d'une interface plus étendue dont la finalité sera de corréler l'intégralité du corpus de la Chronique avec les différents matériaux qui lui sont associés. Au sens large, il s'agit d'une base évolutive qui accueillera toute information en relation avec la Corse médiévale et que le texte de Giovanni della Grossa a possiblement évoquée ${ }^{30}$ (fig. 3). 
Fig. 3 - L'interface graphique de la plateforme d'édition de la Chronique (UMR 6240 LISA).

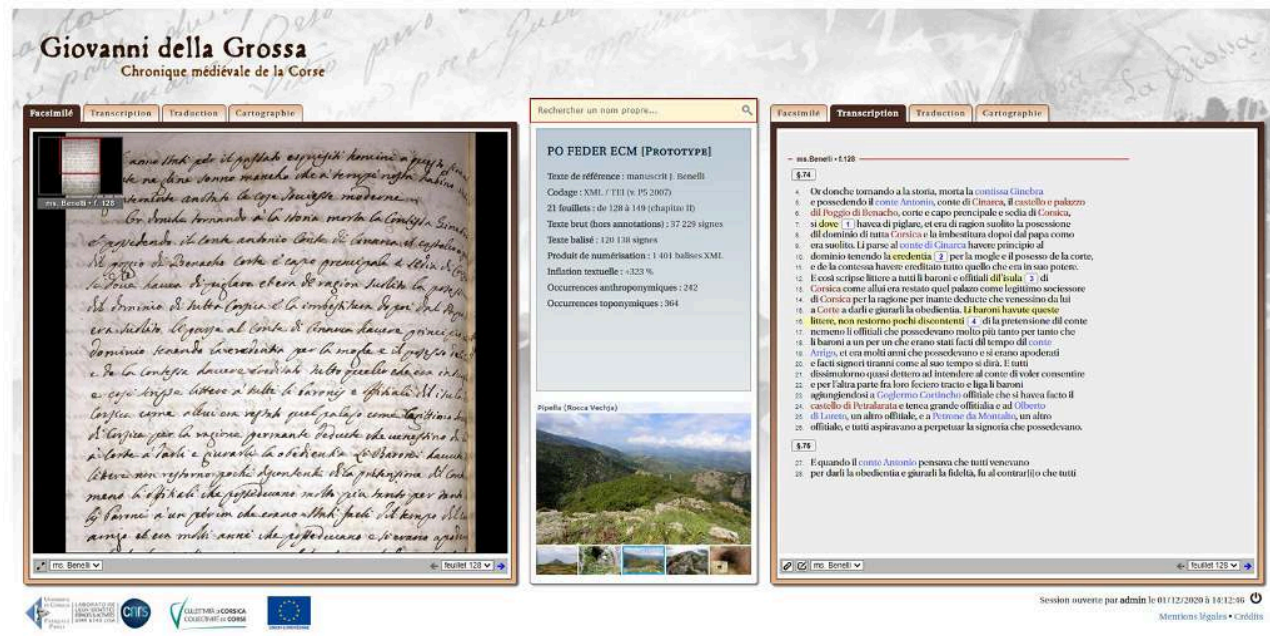

\section{La confrontation du texte aux données archivistiques et archéologiques}

9 La confrontation systématique du récit de Giovanni della Grossa aux documents d'archives et aux données archéologiques doit non seulement permettre d'élaborer l'apparat critique, mais également de renouveler les connaissances sur la Corse médiévale. Un premier programme d'enquête a été lancé en janvier 2018 à Pise ${ }^{31}$, à Gênes $^{32}$, et à Barcelone ${ }^{33}$. Ces dépouillements ont permis d'enrichir la documentation à notre disposition sur les relations économiques entre la Corse et la péninsule italienne ainsi que sur l'histoire politique de l'île aux XIII ${ }^{\mathrm{e}}-\mathrm{XV}^{\mathrm{e}}$ siècles. Ces documents permettent de préciser et parfois de nuancer les propos du chroniqueur. Un corpus significatif de lettres de seigneurs corses aux souverains aragonais, écrites entre la fin du xiII et le milieu du Xve siècle, a notamment pu être constitué (fig. 4). 
Fig. 4 - Lettre de Vincentello d'Istria à Alphonse V d'Aragon du 17 mai 1417.

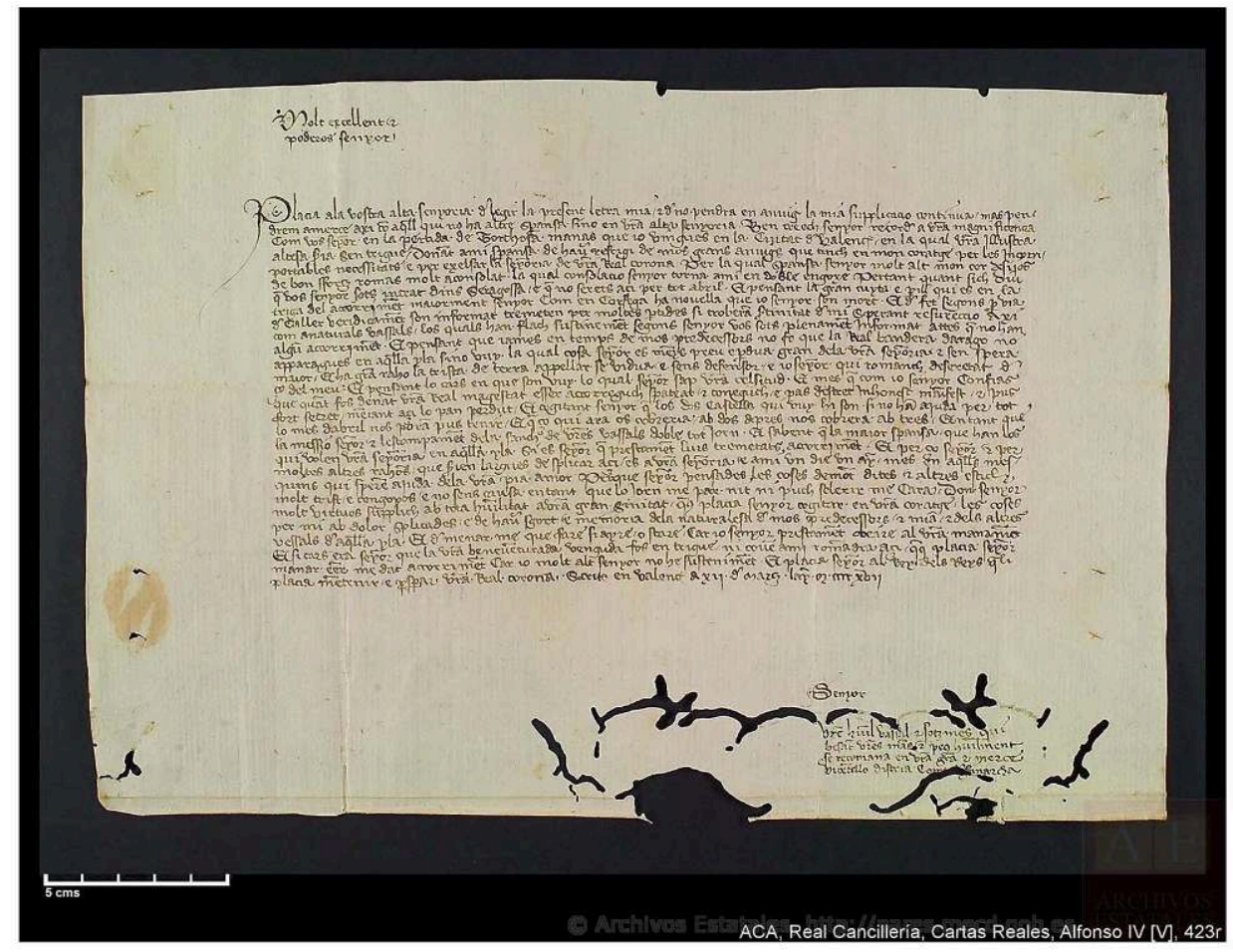

Cl. Archives de la couronne d'Aragon [http://pares.mcu.es/ParesBusquedas20/catalogo/show/ 3182160]

Parmi les éléments structurants le récit de Giovanni della Grossa figure la légende d'une reconquête chrétienne de l'île opérée au $\mathrm{IX}^{\mathrm{e}}$ siècle par le comte romain Ugo Colonna et son allié le comte de Barcelone. Ce mythe constitutif de la légitimité des seigneurs cinarchesi, héritiers d'Ugo Colonna et de son fils Cinarco, est la clef de voûte du discours du chroniqueur. Or, sa fixation définitive sous la plume de Giovanni della Grossa au $\mathrm{Xv}^{\mathrm{e}}$ siècle s'est nourrie à plusieurs sources et renvoie à différentes strates historiques que nous tenterons de reconstituer ${ }^{34}$. Ainsi le premier chapitre de la Chronique sera-t-il confronté aux documents sur la Corse publiés dans les Monumenta Germaniae Historica, dont la plupart sont accessibles en ligne, mais également aux chroniques pisanes qui ont pu constituer une source d'information du notaire corse. Mise en relation avec l'alliance de Vincentello d'Istria et d'Alphonse V d'Aragon (1416-1434) ${ }^{35}$, l'intervention du comte de Barcelone en Corse contre les Maures fait en effet écho à l'offensive commune de Pise, de Gênes et de Barcelone contre le souverain musulman des Baléares, Mujāhid de Denia, en $1015^{36}$. Or, des documents attestent de la participation de Corses et de Sardes à cette offensive chrétienne dont le souvenir a pu alimenter les imaginaires locaux ${ }^{37}$. Un second programme d'enquête, initié en 2019, vise à réaliser des sondages ciblés dans les Archives Vaticanes, qui ont été, jusqu'à ce jour, assez peu exploitées par les chercheurs $\operatorname{corses}^{38}$. Ces dépouillements permettront de mieux saisir la politique pontificale en Corse aux $\mathrm{XIII}^{\mathrm{e}}-\mathrm{XV}^{\mathrm{e}}$ siècles. À cet égard, l'intervention d'Eugène IV dans les années 1440 fera l'objet d'une attention particulière ${ }^{39}$. Envoyé comme ambassadeur des insulaires à Rome, Giovanni della Grossa joua en effet un rôle prépondérant dans cette ultime tentative pour imposer le gouvernement de l'Église en Corse. 
De son côté, l'enquête archéologique s'articule dans un premier temps sur l'analyse des châteaux et des tours mentionnés dans la Chronique. La pertinence de ce choix de sites est renforcée par l'absence notoire d'inventaire des fortifications à l'échelle de la Corse pour cette période. En effet, en dehors des travaux universitaires menés par Gilles Giovannangeli dans les années $1990^{40}$ et ceux de Daniel Istria dans le courant des années $2000^{41}$, ainsi que la réalisation d'une poignée d'interventions archéologiques programmées ${ }^{42}$, la confrontation entre les fortifications mentionnées par Giovanni della Grossa et celles identifiées sur le terrain n'a été que peu exploitée ${ }^{43}$. Pourtant, cette corrélation permet de préciser le nombre de sites et d'en apprécier la nature. La Chronique mentionne, en effet, les fortifications tout à la fois par les termes de castrum, castellu ou encore de rocca. Ainsi, derrière cette terminologie, sont répertoriés sur le terrain à la fois le château, la résidence seigneuriale, le château secondaire, et les tours isolées qui sont des relais de l'autorité seigneuriale (fig. 5 et 6 ).

Fig. 5 - Tour de Crocano à Sartène (cl. É. Tomas, UMR 6240 LISA).

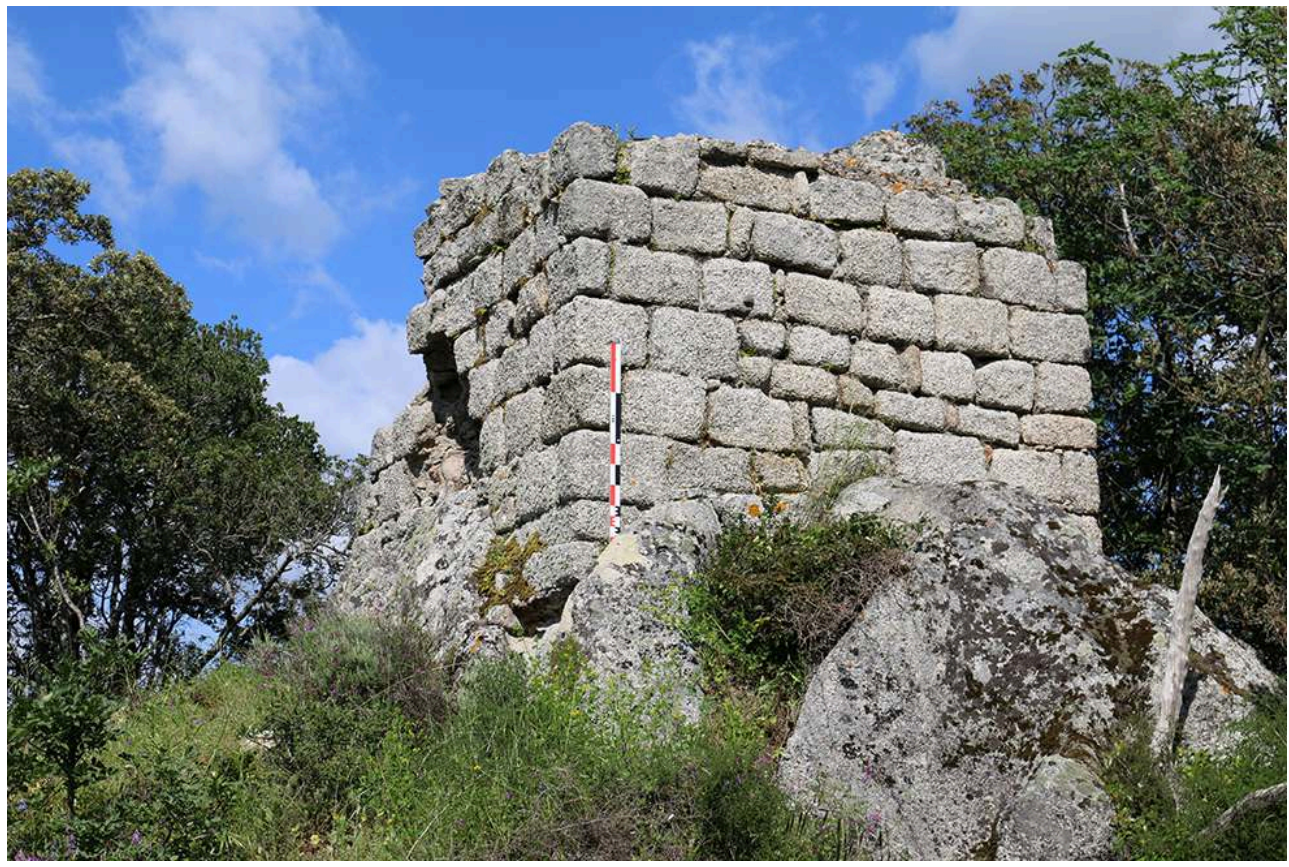


Fig. 6 - Logis du château de Baricini à Sartène (cl. É. Tomas, UMR 6240 LISA).

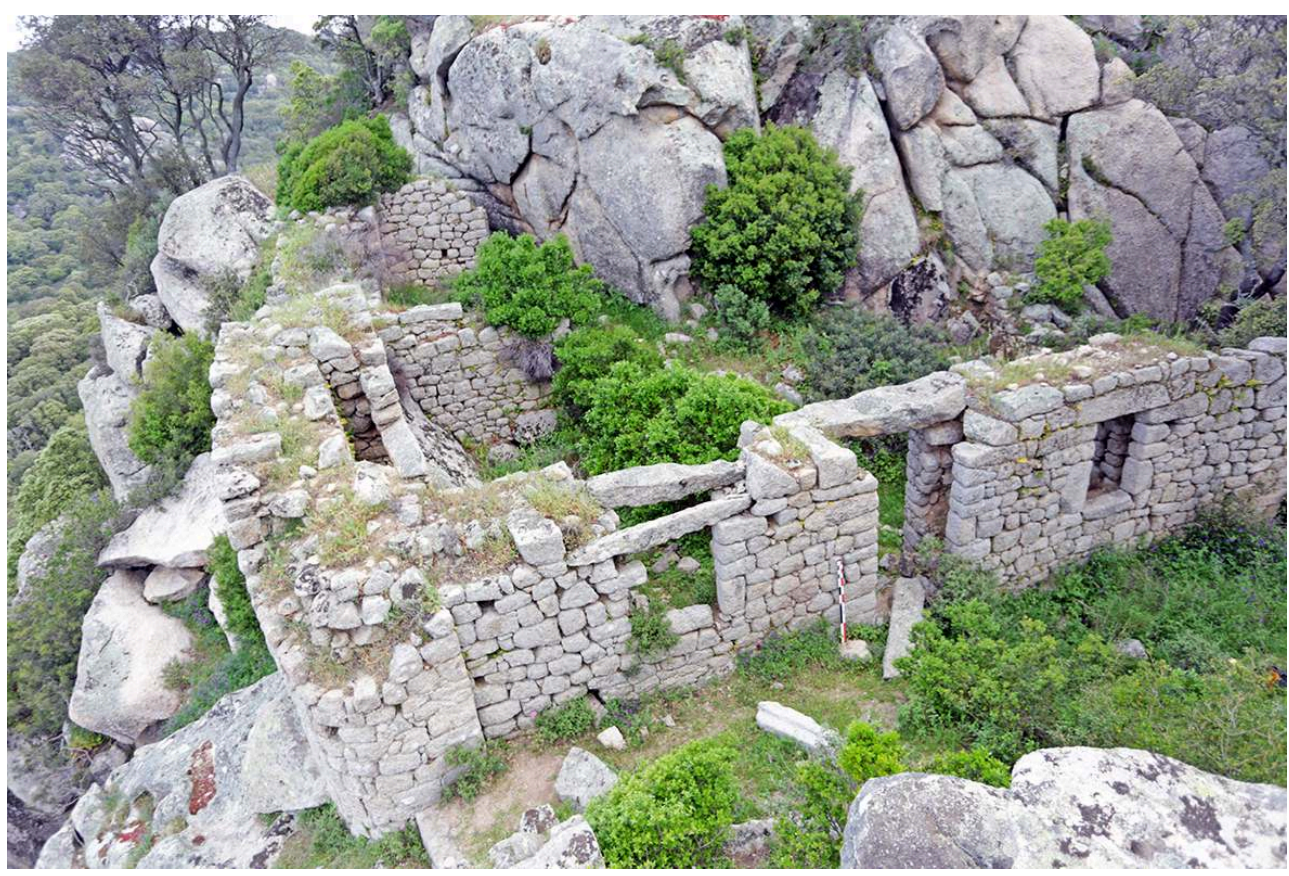

12 Cette identification systématique des entités aboutira in fine à la constitution d'un corpus bénéficiant à la fois d'un support cartographique, accessible en ligne, et d'une base de données archéologiques, avec accès réglementé, essentiels pour aborder le fonctionnement des réseaux défensifs médiévaux de l'île ${ }^{44}$. Cette méthode de traitement insère ainsi chaque site dans son contexte sociopolitique et au sein du réseau dont il dépend.

Dans cette perspective, en 2018, une première étude de terrain a concerné un échantillon d'une soixantaine de sites sur un corpus de cent treize monuments fortifiés mentionnés par Giovanni della Grossa. Ce travail a donné lieu à la production systématique de fiches de sites, reliées à une base de données géo-référencées et à une photothèque légendée. Au terme du traitement de ce premier corpus, une sélection de photographies accompagnée d'une note succincte sera insérée à l'édition numérique avant la fin 2021 (fig. 7). 
Fig. 7 - L'intégration des données archéologiques (UMR 6240 LISA).

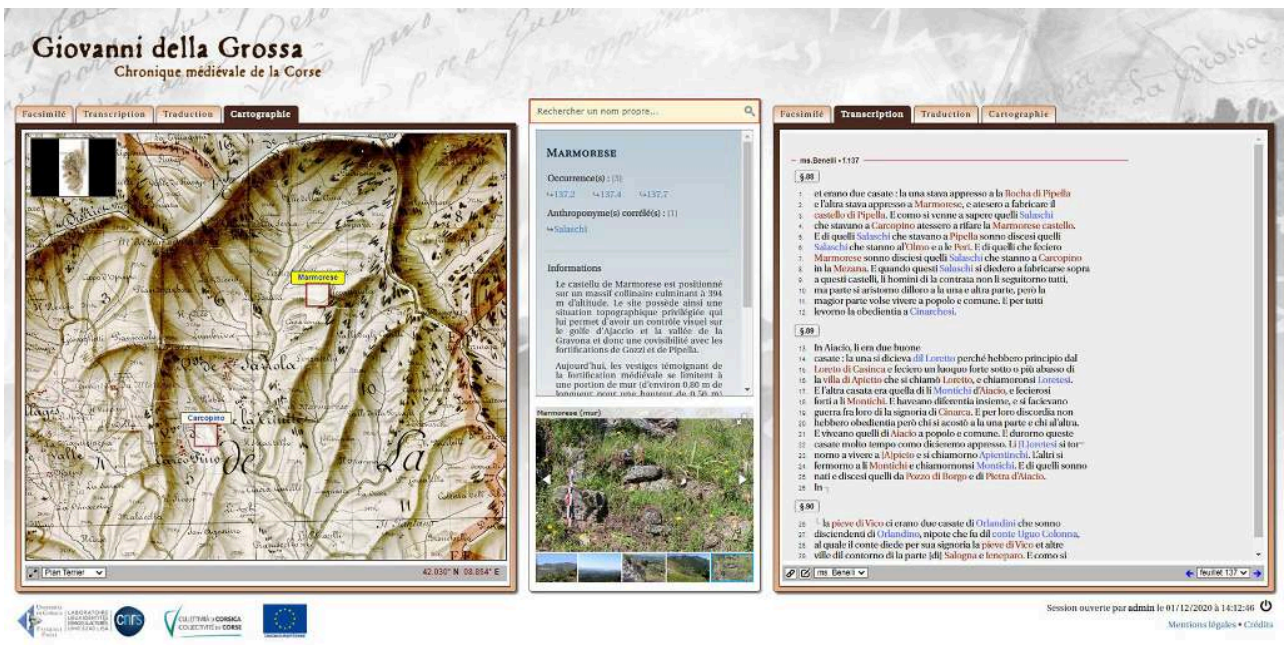

Dans un second temps, les investigations de terrain seront poursuivies dans l'optique de traiter l'intégralité des sites fortifiés mentionnés par Giovanni della Grossa et des recherches seront entreprises sur la vingtaine d'édifices de culte signalés par le chroniqueur ${ }^{45}$.

\section{Constitution d'une base de données prosopographiques}

15 La Chronique de Giovanni della Grossa se présente comme une approche généalogique de la société corse au $\mathrm{XV}^{\mathrm{e}}$ siècle ${ }^{46}$. Elle s'étend complaisamment sur les ascendances, sur les liens de parenté et sur les alliances familiales des lignées seigneuriales ou celles des chefs populaires ${ }^{47}$. Comme dans l'ensemble de l'Occident médiéval, l'affirmation d'un passé prestigieux est une obligation pour tous ceux qui veulent légitimer leur domination sociale. Cependant, le passé ne suffit pas à cimenter de façon durable les liens entre les membres des groupes dominants et les alliances doivent continuellement être réactivées. Ces liens familiaux constituent l'ossature la plus solide des réseaux humains, denses et étendus, qui quadrillent l'île et qui parfois s'élargissent à une partie de la Méditerranée occidentale ${ }^{48}$. La constitution d'une base de données prosopographiques permettra de générer des arbres généalogiques ainsi que des cartes dynamiques replaçant les individus dans leur contexte familial et leurs réseaux d'alliés et d'ennemis ${ }^{49}$ (fig. 8 et 9). 
Fig. 8 - Le réseau d'alliés de Vincentello d'Istria en 1426 selon Giovanni della Grossa (DAO M. Ferrara).

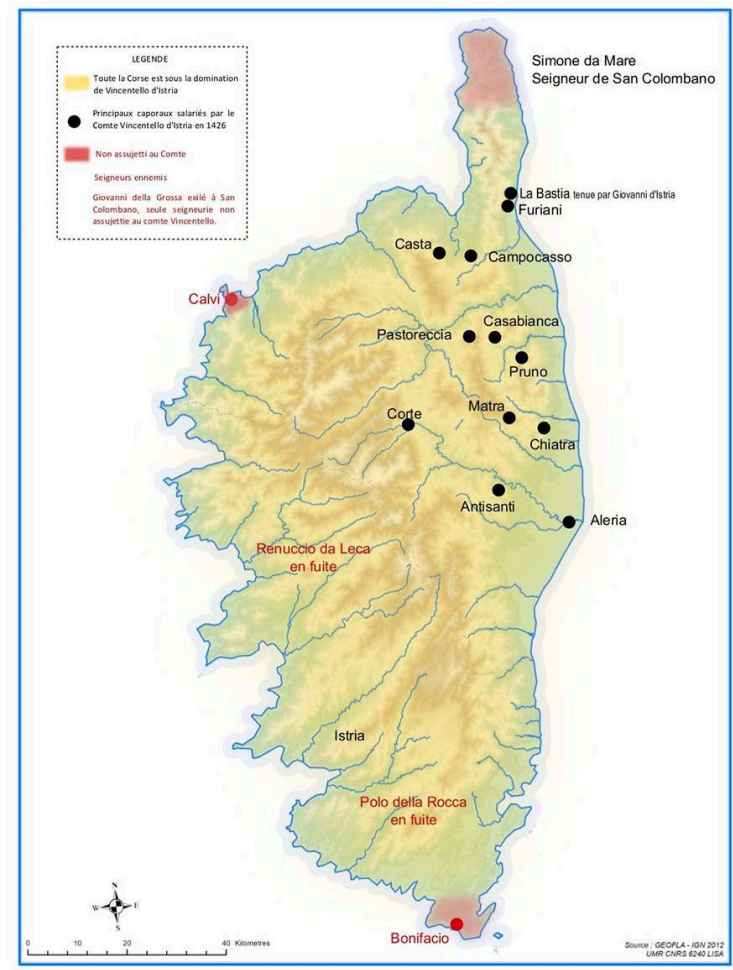

Fig. 9 - La rétraction du réseau de Vincentello d'Istria en 1430 selon Giovanni della Grossa (DAO M. Ferrara).

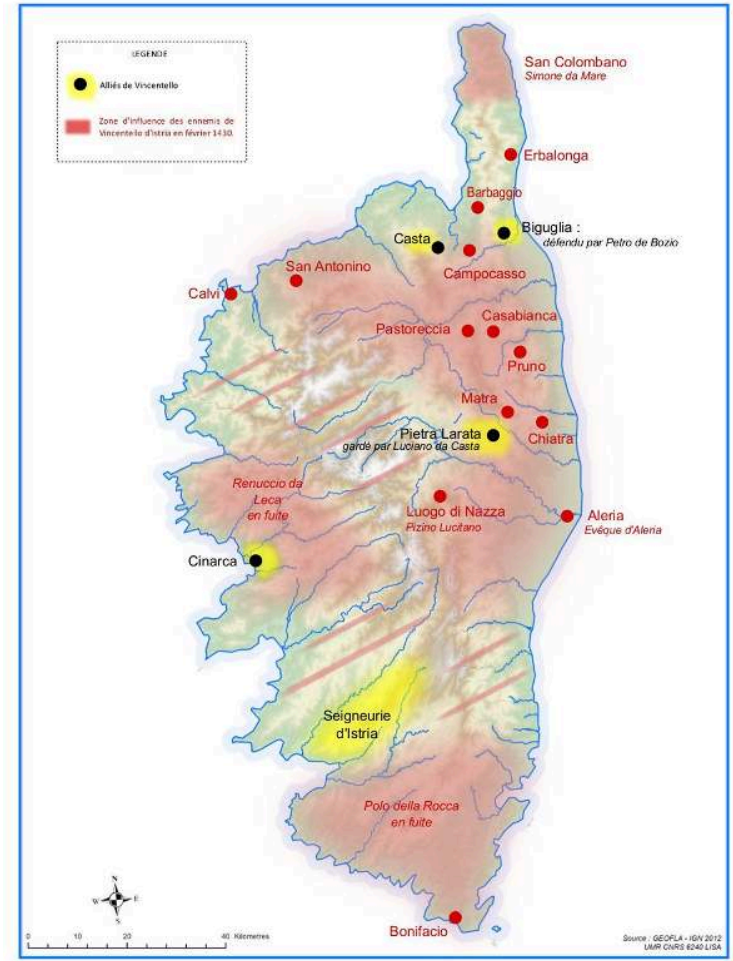


Tout en soulignant les parcours individuels, l'enquête prosopographique éclairera la composition des groupes sociaux et mettra en lumière les conditions qui favorisent l'accès au pouvoir. Le chroniqueur brosse, en effet, un tableau de la Corse du $\mathrm{xv}^{\mathrm{e}}$ siècle qui ne se borne pas au monde seigneurial. Il évoque longuement la catégorie des officiers qui jouent un rôle primordial dans la vie publique et qui participent à la genèse de l'État moderne dans l'île. Son texte permet d'entrevoir la polyvalence des charges dévolues aux personnels qui contribuent à la gouvernance des territoires. Notaires, vicaires, châtelains, commissaires, intendants, messagers ou ambassadeurs, tous évoluent dans la sphère du pouvoir et s'y maintiennent quelquefois pendant des décennies. Là encore, l'auteur démontre l'importance des liens familiaux, que les officiers soient des parents proches des seigneurs, ou que leurs charges soient transmises de façon héréditaire. À travers l'analyse de ses écrits se dessinent donc la constitution et l'évolution des élites sociales de la Corse médiévale.

\section{Conclusion}

17 En proposant une nouvelle édition de la Chronique de Giovanni della Grossa au format numérique, notre ambition est d'apporter une réponse innovante aux problèmes que soulève l'écriture de l'histoire d'un territoire insulaire et peu documenté tel que la Corse. En premier lieu, il s'agit d'un projet d'histoire globale et connectée qui, s'il a pour point de départ la Corse, vise à éclairer une partie de l'histoire de la Méditerranée occidentale : l'imaginaire des relations entre islam et chrétienté; l'affirmation des puissances économiques de Pise et de Gênes; la lutte entre guelfes et gibelins; les visées pontificales sur l'espace tyrrhénien; les impérialismes catalans et génois. Dans un contexte d'extrême dispersion des documents d'archives et de rareté des études archéologiques, le support numérique présente, en effet, l'avantage de faciliter considérablement le croisement des sources, les jeux d'échelle et la collaboration entre les disciplines: histoire, philologie, archéologie, histoire de l'art, linguistique et informatique. En adossant ce projet d'histoire totale au texte de Giovanni della Grossa, nous souhaitons non seulement valoriser la Chronique à destination de la communauté scientifique comme du public, mais également tenter de répondre à un certain nombre de questions cruciales que ce récit historique soulève: quelle était l'ambition de Giovanni della Grossa lorsqu'il entreprit son œuvre? Quelles ont été ses sources? Peuton y voir une esquisse de construction nationale autour d'une dynastie (les Cinarchesi) et d'un territoire (la Corse) ${ }^{50}$ ? Y a-t-il des traces de formes orales corses du Xve siècle dans ce texte écrit en italien? Pourquoi cette chronique a-t-elle été si peu diffusée ? En quoi les réécritures successives ont-elles volontairement brouillé la compréhension du discours originel du chroniqueur?

18 Au-delà de l'intérêt patrimonial et historique de la restitution d'un texte fondateur de l'historiographie et de la littérature corses, qui s'inscrit dans le contexte plus général de la chronachistica italienne, l'édition numérique de Giovanni della Grossa constitue, à l'échelle de l'île, une expérience pionnière de plateforme de recherche collaborative ${ }^{51}$ : elle permettra de décloisonner les travaux afin d'écrire collectivement une histoire méditerranéenne de la Corse $\mathrm{e}^{52}$. À l'heure où le modèle de la recherche sur projet tend à s'imposer en France, en entraînant une raréfaction des postes, l'édition numérique de Giovanni della Grossa se présente en outre comme une opportunité de maintenir le dynamisme de la discipline médiévale à l'université de Corse ${ }^{53}$. 
Reçu : 30 août 2020 - Accepté : 16 novembre 2020

\section{NOTES}

1. Depuis le début des années 2000, une dizaine de thèses en histoire et archéologie médiévales ont été réalisées à l'université de Corse sous la direction de Jean-André Cancellieri : A. FRANZINI, Politique et société: la Corse du Quattrocento, thèse de doctorat d'histoire médiévale, sous la direction de J.-A. Cancellieri, université de Corse, 2003 [Politique et société : la Corse du Quattrocento, Ajaccio, Piazzola, 2005]; V. MARCHI VAN CAUWELAERT, Recherches sur l'organisation territoriale génoise dans les seigneuries du sud de la Corse (milieu Xve-début XVI siècle), thèse de doctorat d'histoire médiévale, sous la direction de J.-A. Cancellieri et la codirection d'É. Crouzet-Pavan, université de Corse, 2007 [La Corse génoise. Saint Georges, vainqueur des «tyrans » (milieu XVe-début XVI siècle), Paris, Classiques Garnier, 2011] ; É. TomAs, Contribution à l'étude de l'habitat rural de la fin du Moyen Âge: caractérisation des dynamiques de peuplement de la pieve de Rostino en haute Corse, thèse de doctorat d'archéologie médiévale, sous la direction de J.-A. Cancellieri et la codirection de D. Istria, université de Corse, 2010 ; P. CAMUFFO, Gli edifici medievali nella valle del Golo: analisi archeologica sull'evoluzione delle tecniche murarie in pietra tra XI e XVI secolo, thèse de doctorat d'archéologie médiévale, sous la direction de J.-A. Cancellieri et la codirection de D. Istria, université de Corse, 2012 ; D. Broc, Dynamiques politiques, économiques et sociales dans la Corse médiévale: le diocèse de Nebbio ( $\left.\mathrm{XI}^{e}-\mathrm{c} .1540\right)$, thèse de doctorat d'histoire médiévale, sous la direction de J.-A. Cancellieri et la codirection de J.-P. Boyer, université de Corse, 2014 ; C. ZEDDA, Dynamiques politiques dans la mer Tyrrhénienne du XI au XII siècle : le rôle de la Sardaigne et de la Corse dans l'espace sous tutelle pontificale, thèse de doctorat d'histoire médiévale, sous la direction de J.-A. Cancellieri et la codirection d'E. Salvatori, université de Corse, 2015 [Ai piedi dell'apostolo. Sede apostolica e spazio tirrenico (secoli XI-XII), Milan, Vita e pensiero, 2020] ; P. ColomBANI, Les Corses et la Couronne d'Aragon: projets politiques et affrontements des légitimités, thèse de doctorat d'histoire médiévale, sous la direction de J.-A. Cancellieri et la codirection de M.-G. Meloni, université de Corse, 2015 [Les Corses et la Couronne d'Aragon: projets politiques et affrontements des légitimités, Ajaccio, Piazzola, 2019]; M. FERRARA, L'espace médiéval de la Corse. Représentations et problématiques autour de la Chronique de Giovanni della Grossa, thèse d'histoire médiévale, sous la direction de J.-A. Cancellieri, université de Corse, 2019.

2. Les quelques chartes monastiques conservées aux Archives départementales de la Haute-Corse ont été publiées dans S. SCALFATI, La Corse médiévale, Ajaccio, 1994. Leur étude peut être complétée par la consultation des documents de l'abbaye de San Venerio del Tino conservés à Turin, voir G. PISTARINo, Le carte del Monastero di San Venerio del tino 1080-1500, Casale, 1944.

3. La présence de la Corse médiévale dans les dépôts d'archives pisans est réelle mais fort irrégulière. L'Archivio arcivescovile contient de nombreux documents dispersés, mais importants sur la présence et l'action ecclésiastiques de Pise dans l'île. L'implantation monastique pisane, notamment celle de l'abbaye de la Gorgone, y est aussi documentée ainsi que dans les archives de la chartreuse de Calci, voir S. ScAlfati, La Corse médiévale..., op. cit. L'Archivio di Stato di Pisa comprend plusieurs fonds qui éclairent l'émigration des Corses à Pise et dans son "contado " (entre autres celui du Comune A). Néanmoins, l'une des sources principales sur les échanges commerciaux entre l'île et Pise et sur l'émigration des Corses à Pise ainsi qu'à Livourne aux XIV et $\mathrm{XV}^{\mathrm{e}}$ siècles est représentée par l'important fonds notarial pisan déposé à l'Archivio di Stato di 
Firenze. Sur cette documentation pisane, voir H. TAVIANI, « Les relations entre la Corse et Pise à la fin du Moyen Âge ", Annales du midi: revue archéologique, historique et philologique de la France méridionale, t. 81 (1969), p. 84-91 ; J.-A. CANCELLIERI, «Pise et la Corse au Moyen Âge », in F. GEMINI (dir.), Corsica e Toscana. Dieci secoli di storia nei documenti pisani e corsi, Pise, 2013, p. 11-19.

4. Pour une orientation générale, voir A.-M. Graziani et A. VentuRINI, Vistighe corse. Guide des sources de l'histoire de la Corse dans les archives génoises, t. 1, vol. 1 (Moyen Âge, 1121-1483), Ajaccio, 2009. Outre la documentation enregistrée dans les Libri Iurium de la ville et publiée par D. PUNCUH (éd.), I libri iurium della Repubblica di Genova, Rome, 1992-1998, quatre fonds principaux de l'Archivio di Stato di Genova éclairent la Corse: les notaires (Notai Antichi, Notai Ignoti), l'Antico Comune, l'Archivio Segreto et le Banco di San Giorgio. Ces fonds ont été analysés dans V. ViTALE, Documenti sul castello di Bonifacio nel secolo XIII, vol. I (LXV della raccolta), fasc. II, Gênes, 1936 ; ID., Nuovi documenti sul Castello di Bonifacio nel secolo XIII, vol. IV (LXVIII della raccolta), fasc. II, Gênes, 1940 ; G. PETTI BALBI, Genova e Corsica nel Trecento, Rome, 1976 ; J.-A. CANCELLIERI, Gênes en Corse et en Sardaigne au XIII ${ }^{e}$ siècle, 4 vol., université d'Aix-Marseille, 1978 ; C. BORNATE, Genova e Corsica alla fine del Medio Evo, Milan, 1940; A. FrANZINI, La Corse du Quattrocento..., op. cit.; V. MARCHI VAN CAUWELAERT, La Corse génoise..., op. cit. ; D. BRoc, Dynamiques politiques..., op. cit.

5. Bien que le Regnum Sardiniae et Corsicae soit demeuré théorique dans la seconde île, de nombreux documents sur la Corse se trouvent dispersés au sein des Archives de la Couronne d'Aragon dans les fonds de la Cancelleria et du Real Patrimonio, voir : A. MARONGIU, «La Corona d'Aragona e il Regno di Corsica ", Archivio Storico di Corsica, t. 4, 1935, p. 481-501; G. SORGIA, Sardenya i Corsega des de la infeudació fins a Alfons "el Magnànim ", Barcelone, 1968; L. D'ARIENZO, Carte reali diplomatiche di Pietro IV il Cerimonioso, re d'Aragona, riguardanti l'Italia, CODOIN, t. 48, Padoue, 1970 ; M.-G. MELONI, « Arrigo della Rocca : un nobile corso al servizio del re d'Aragona », Medioevo. Saggi e rassegne, 18 (1993), p. 9-26 ; EAD., « La corona d'Aragona e la Corsica attraverso una relazione di Castruccio Castracani signore di Lucca », XIV congesso internazionale di storia de la Corona d'Aragona, vol. II, t. 2, Sassari, 1995, p. 595-628 ; M.-T. FERRER I MALLOL, « Il partito filocatalano in Corsica dopo la morte di Arrigo della Rocca ", in M.-G. MELONI (éd.), La Corsica e il mondo mediterraneo nell'età medioevale, éd. Medioevo. Saggi e rassegne, 24 (2001), p. 65-88; P. Colombani, Les Corses et la Couronne d'Aragon..., op. cit.

6. Voir J.-A. CANCELLIERI, « Giovanni della Grossa », in Dizionario Biografico degli Italiani, t. 37, Rome, 1989 ; A. FRANZINI, «Giovanni della Grossa, la carrière d'un notaire en Corse au Quattrocento », Cahiers Corsica, 2003.

7. Voir V. MARCHI VAN CAUWELAERT, La Corse génoise..., op. cit., p. 343-346.

8. Voir M. ZABBIA, I notai e la chronachistica cittadina italiana nel Trecento, Rome, 1999.

9. Ce choix s'est en partie inspiré de l'édition du Petit Thalamus de Montpellier, en ligne [http:// thalamus.huma-num.fr/].

10. Le projet prévoit d'intégrer la copie numérique des trois manuscrits principaux de la Chronique afin d'en faciliter la comparaison. Les manuscrits $\mathrm{Y}$ et $\mathrm{BnF}$, étant déjà en ligne, pourront être consultés librement, tandis que la consultation du Benelli, soumis à des droits, sera réservée aux seuls chercheurs. En cas de variantes, les liens hypertextes permettront de passer directement de l'annotation philologique aux photos des pages des manuscrits concernés.

11. À l'Archivio di Stato di Genova, voir les lettres du Doge à Galeazzo de Campofregoso à propos de Giovanni della Grossa, en 1449 (Archivio segreto, section Litterarum, Registri dei cancellieri, 1791, années $1447-1455,1457, \mathrm{n}^{\circ} 589$, fol. $156 \mathrm{v}^{\circ}$ et $\mathrm{n}^{\circ} 592$, fol. $157 \mathrm{v}^{\circ}$ ) ; la lettre de Giovanni della Grossa aux Protecteurs de Saint-Georges en 1456 (Banco di San Giorgio, section Primi cancellieri di San Giorgio, liasse 40, $\mathrm{n}^{\circ}$ 363, fol. 362-363).

12. R. COLONNA DE CESARI ROCCA, « Un ministre de Philippe II auteur d'une Histoire de la corse (Matheo Vázquez de Leca) », Revue historique, 126 (1917), p. 1-11 ; A. TRAMONI, Histoire d'un livre : le manuscrit de Bastia de la chronique de Giovanni della Grossa, catalogue d'exposition, Bastia, 2015 ; L. ARRIGHI, 
Pouvoir et historiographie : les Histoires de Corse ( $\mathrm{XV}^{e}-\mathrm{XVI} \mathrm{e}^{e}$ siècles) entre France, Italie et Espagne, thèse de doctorat, sous la direction de G. Martin, Sorbonne Université, 2019.

13. L. Letteron (éd.), Le Croniche di Giovanni della Grossa e di Pier' Antonio Monteggiani, Bastia, 1910.

14. L'ancien propriétaire était membre de la Société des sciences historiques et naturelles de la Corse, tout comme l'abbé Letteron. N'ayant pu consulter ce manuscrit, nous nous fondons sur la description qu'en fait Antoine-Marie Graziani dans l'édition de 2016.

15. A. Casanova et M. giacomo-Marcellesi (éd.), Chronique médiévale corse de Giovanni della Grossa, Ajaccio, 1999.

16. La question de l'origine du manuscrit Benelli soulève des difficultés d'interprétation. Il se pourrait qu'il s'agisse du manuscrit Pozzo di Borgo, qui avait été vendu dans les années 1980 et qui a disparu depuis lors. Certes, ce manuscrit avait été écarté par Letteron en raison de sa graphie modernisée, mais la reliure romantique du XIX siècle du manuscrit Benelli est complexe en ce sens qu'elle comprend plusieurs cahiers rédigés par des mains diverses à des moments différents. Si le premier cahier, ajouté pour combler un manque, montre bien une graphie modernisée, les cahiers suivants sont plus anciens. Nous pouvons donc émettre l'hypothèse que l'abbé Letteron se serait contenté de ces premières pages modernes pour écarter ce manuscrit de l'édition au profit du manuscrit $Y$ jugé plus exploitable en raison de ses qualités graphique et calligraphique, dans un ensemble soigné et homogène de surcroît.

17. A.-M. Graziani (éd.), Giovanni della Grossa, Pier'Antonio Montegiani. Chronique de la Corse des origines à 1546, Ajaccio, 2016.

18. Les précédentes éditions ont supprimé les passages jugés, à tort ou à raison, modernes afin de proposer un texte plus proche de la version originale. Le choix d'un support numérique nous permet de conserver les interpolations en les signalant au moyen d'une balise spécifique et d'une annotation détaillée afin de préserver l'intégrité du manuscrit et de rendre visibles les réécritures successives. Ces dernières pourront ainsi faire l'objet d'analyses ultérieures de la part d'historiens modernistes.

19. L. ARRIGHI, Pouvoir et historiographie..., op. cit.

20. À deux reprises le compilateur attribue des passages au chroniqueur Marc'Antonio Ceccaldi, ostensiblement pro-génois. Cependant, le manuscrit de la BnF et le Galeazzini mentionnent le nom du continuateur de Giovanni della Grossa, Pier' Antone Montegiani, moins marqué par son appartenance politique. Ces changements d'attributions sont certainement motivés par les engagements politiques des auteurs et des copistes, lesquels ne sont plus favorables à Gênes, voir L. ARRIGHI, Pouvoir et historiographie..., ibid.

21. À titre d'exemple, alors que les manuscrits Benelli et $\mathrm{Y}$ ne précisent pas le nom d'un château dans l'Ortolo (Sartène), le copiste du manuscrit de la BnF ajoute le nom de Fontanella.

22. Text Encoding Initiative [https://tei-c.org/]. Ce choix a été fait au terme d'une formation dispensée par l'École nationale des chartes au printemps 2019, car il répondait aux besoins du projet tout en étant adapté aux compétences informatiques des membres de l'équipe. Le schéma adopté repose sur un nombre limité de balises : 1) la segmentation du texte ;2) les toponymes ; 3 ) les anthroponymes; 4) les interpolations; 5) les apostilles; 6) les insertions documentaires réalisées par les copistes successifs. Ce schéma pourra toutefois être développé afin de répondre à de nouveaux besoins nés de l'avancée des travaux.

23. L'échantillon choisi concerne le chapitre de la Chronique dédié à l'émergence féodale qui suit l'assassinat du comte légendaire Arrigo Bel Messer, aux alentours de l'an Mil. Ce choix permettait, en effet, de construire les différents éléments de la base et, notamment, le volet archéologique bien représenté dans l'extrait.

24. Il sera, par exemple, possible de confronter le récit de Giovanni della Grossa sur l'action de Vincentello d'Istria (1402-1434) aux lettres de ce dernier conservées aux Archives de la Couronne d'Aragon et accessibles en ligne sur le portail des archives espagnoles [http:// pares.culturaydeporte.gob.es/inicio.html]. 
25. Les deux panneaux latéraux permettent de mettre en regard le manuscrit et la transcription ou encore la transcription et la traduction. Le module central permet quant à lui d'enrichir l'annotation du texte.

26. Par exemple : « règle des trois clics », intuitivité de la navigation, compatibilité des supports, etc. [https://www.w3.org/WAI/].

27. Projet JavaScript OpenSeaDragon [https://openseadragon.github.io/].

28. Archives départementales de Corse-du-Sud, 1 C, Plan Terrier de la Corse, 1770-1795.

29. Tous les toponymes mentionnés dans le texte sont géo-référencés sur le Plan Terrier et sur la carte IGN au $1 / 25000^{\mathrm{e}}$.

30. Initié dans le cadre du programme FEDER 2018-2021, ce projet de recherche a vocation à être pérennisé dans le temps. Des discussions sont en cours pour un hébergement du projet sur la plateforme Huma-Num. L'année 2021 sera consacrée à l'achèvement de la transcription et de l'encodage du texte assorti d'une première traduction et annotation dans la perspective d'une mise en ligne début 2022. L'annotation sera par la suite constamment enrichie par de nouvelles recherches philologiques, archivistiques, archéologiques et linguistiques, afin d'aboutir à une véritable encyclopédie numérique sur la Corse médiévale.

31. Outre la documentation notariée, les recherches à l'Archivio di Stato di Pisa ont permis d'examiner les chroniques inédites conservées dans le fonds Roncioni.

32. Les dépouillements concernent les fonds de l'Archivio Segreto et du Banco di San Giorgio.

33. Les recherches ont porté essentiellement sur les fonds de la Cancelleria, mais des sondages complémentaires sont prévus dans le fonds du Real Patrimonio.

34. Un document du XIV ${ }^{\mathrm{e}}$ siècle, conservé à Pise, édité par Pär Larson, témoigne que des traditions relatives à des comtes de Corse circulaient déjà à cette époque. En outre, on y trouve la mention du toponyme " poio del palazo ", qui renvoie dans le récit du chroniqueur au palais des premiers comtes carolingiens à Corte, voir P. LARSON, « Note su un dossier di falsi documenti corsi copiati nel 1364 », Atti del VI Congressi degli Italianisti Scandinavi (16-18 agosto 2001), Lund, 2003, p. 325-339.

35. Voir V. MARCHI VAN CAUWELAERT, « Seigneurie contre Commune. Recherche sur les fondements idéologiques du parti aragonais en Corse ", Mélanges de la Casa de Velázquez, 43/1, 2013, p. 231-249. 36. Émir de Denia, Mujāhid avait étendu son pouvoir sur les îles Baléares et la Sardaigne, cf. T. BRUCE, La taïfa de Denia et la Méditerranée au XI siècle, Toulouse, 2013, p. 145-191. Giovanni della Grossa fait allusion à ces événements à l'issue de son récit de la « reconquête » de la Corse pour évoquer la Sardaigne voisine.

37. C. CAlisSe (éd.), Liber Maiolichinus de gestis Pisanorum illustribus. Poema della Guerra Balearica Secondo il Cod. Pisano Roncioni, Rome, 1904 ; M.-T. FERRER I MALLoL et M. RIU I RIU (dir.), Tractats $i$ negociacions diplomàtiques de Catalunya $i$ de la Corona catalanoaragonesa a l'edat mitjana, vol. I/1 (Tractats i negociacions diplomàtiques amb Occitània, França i els estats italians 1067-1213), Barcelone, 2009, p. 289 sqq. Nous remercions Stéphane Péquignot de nous avoir indiqué ce document.

38. De premières recherches sur les $\mathrm{XI}^{\mathrm{e}}$-XII ${ }^{\mathrm{e}}$ siècles avaient été entreprises par Corrado Zedda dans le cadre de sa thèse de doctorat, voir C. ZEDDA, Ai piedi del apostolo..., op. cit. Une mission réalisée en 2019 a permis de poursuivre l'analyse de la politique pontificale au XIII ${ }^{\mathrm{e}}$ siècle. Ces recherches, interrompues en 2020 en raison de la crise sanitaire, reprendront en 2021.

39. En 1445, le pape Eugène IV envoya un gouverneur pontifical dans l'île. Selon Giovanni della Grossa, le pape répondait ainsi à la demande des insulaires qui, considérant que l'île faisait partie du patrimoine de Saint-Pierre, lui avait envoyé plusieurs ambassades. À ce jour, outre le registre de l'évêque de Potenza (gouverneur pontifical), édité dans L. SANDRI, «Il governo pontificio in Corsica all'epoca di Eugenio IV », Archivio Storico di Corsica, 15 (1937), p.1-26, seuls deux documents sont connus : Archivio Segreto Vaticano, Mandati Camerali, registre $n^{\circ} 830$, fol. $149 \mathrm{r}^{\circ}$ et fol. $166 \mathrm{v}^{\circ}$, le seigneur Vincentello d'Istria y obtient des subsides de la cour pontificale (décembre 1445); Archivio Segreto Vaticano, Registri vaticani 383 , fol. $3 v^{\circ}$ et $31 r^{\circ}-v^{\circ}$, Giovanni della Grossa y 
obtient l'office de greffier de justice de Corse (20 octobre 1445), voir A. FRANZINI, « Giovanni della Grossa, la carrière d'un notaire... ", op. cit., p. 14-15.

40. G. GiovanNANGELI, « Recherches sur les castelli cinarchesi à la fin du Moyen Âge (1340-1505) », Bulletin de la Société des sciences historiques et naturelles de la Corse, 659 (1991), p. 99-123.

41. D. IsTRIA, Châteaux et habitats fortifiés dans le nord de la Corse (1077-1358), thèse de doctorat, sous la direction de M. Fixot, université de Provence, 2000 [Pouvoirs et fortifications dans le nord de la Corse, $\mathrm{XI}^{e}$-XIV ${ }^{e}$ siècle, Ajaccio, 2005].

42. À ce jour, un peu plus d'une vingtaine de fortifications ont fait l'objet d'investigations qui ont rarement dépassé le stade du sondage de superficie réduite. Seul le castrum de Rostino (Castellodi-Rostino) a été étudié dans sa quasi intégralité durant une dizaine d'années sous la direction de D. Istria (CNRS).

43. Cent treize sites sont mentionnés par Giovanni della Grossa qui évoque : le contexte de leur édification, de leur destruction et de leur reconstruction; les événements qui s'y sont déroulés (sièges, naissances, complots et assassinats); leurs différents possesseurs. Son texte permet également d'opérer une première hiérarchisation des châteaux en fonction de leur importance symbolique et politique: le palazzu de Venaco (Corte), siège du pouvoir des premiers comtes légendaires ; Cinarca (Casaglione), capitale de la Corse seigneuriale ; San Colombano (Rogliano), centre d'un commerce actif entre l'île et l'Italie ; Biguglia (Lucciana), siège du pouvoir génois ; Leca (Arbori), Ornano (Urbalacone), Bozzi (Guitera-les-Bains), la Rocca (Olmeto), Litala (Sartène), les châteaux éponymes des seigneurs du sud de l'île. Ces indications sont d'autant plus précieuses que les sources d'archives font défaut, à l'exception de quelques inventaires génois de la fin du $\mathrm{XV}^{\mathrm{e}}$ siècle, voir A.-M. GRAZIANI, «Étude des inventaires de châteaux de Cinarca (Zurlina, Cinarca, Leca et Sia) 1450-1500: une aide à l'archéologie ", in Patrimoine d'une île: recherches récentes d'archéologie médiévale en Corse, Ajaccio, 1995, p. 97-103.

44. Cette base de données sur les fortifications viendra enrichir la carte archéologique de Corse (DRAC de Corse). Son accès sera soumis à autorisation afin d'éviter les opérations de fouilles sauvages qui sont malheureusement fréquentes dans l'île.

45. Giovanni della Grossa mentionne la construction des cathédrales de Mariana, de Nebbio, d'Accia et d'Aleria, ainsi que celle de l'église de Saint-Jean à Corte, située à proximité du palazzu de Venaco. Il évoque également plusieurs églises comme lieux de sépultures aristocratiques: c'est le cas notamment de l'église de Santo Spirito à Olmeto, où serait enterré le premier comte de Corse, Arrigo della Rocca, et de l'église de Frasso à Cauro, où serait inhumé le vice-roi de Corse Jaime de Besora. Ces indications pourraient permettre de programmer des sondages archéologiques afin de faire progresser les connaissances sur les rites funéraires aristocratiques. Sur cette question, voir A.-G. CORBARA, Sépultures et pratiques funéraires en Corse au Moyen Âge ( $v^{e}$ $X V^{e}$ siècles) : première approche archéo-anthropologique à partir des fouilles récentes des sites d'Ajaccio, Mariana et Sagone, thèse de doctorat d'archéologie médiévale, sous la direction D. Istria, R. Martorelli et M. Signoli, université d'Aix-Marseille, 2016.

46. Voir F. ETTORI, La Maison de la Rocca. Un lignage seigneurial en Corse au Moyen Âge, Ajaccio, 1998.

47. Voir M. FERRARA, L'espace médiéval de la Corse..., op. cit.

48. Outre la tradition légendaire rattachant la noblesse insulaire aux grandes familles romaines, le chroniqueur évoque aux $\mathrm{XII}^{\mathrm{e}}$-XIII ${ }^{\mathrm{e}}$ siècles, des alliances matrimoniales entre les seigneurs corses, les juges sardes et l'aristocratie pisane, qui ne sont pas avérés par la documentation d'archives, mais reflètent des alliances politiques bien réelles, voir V. MARCHI VAN CAUWELAERT, Giudice di Cinarca et Mariano d'Arborea. À l'origine du parti aragonais en Corse et en Sardaigne, Mélanges de la Casa de Velázquez, à paraître. À partir du XIV siècle, les informations de Giovanni della Grossa se font plus précises et concernent des unions entre la noblesse corse et l'aristocratie génoise, les Adorno et les Doria notamment. Les archives de la Couronne d'Aragon témoignent quant à elle 
d'un projet avorté de mariage entre le comte Vincentello d'Istria et une aristocrate catalane en 1413.

49. L'ensemble du corpus demeure assez restreint et se limite à une centaine d'individus. Chaque individu fera l'objet d'une fiche biographique insérée en note afin d'aboutir à un dictionnaire prosopographique. À partir des éléments fournis par la Chronique et les sources d'archives, chaque individu sera mis en relation avec ses ascendants, ses alliés, ses ennemis, des lieux qui lui sont attachés et des fonctions qu'il a exercées. Afin de représenter l'extrême volatilité des alliances et le caractère lacunaire des sources qui accroît la marge d'incertitude, la durée sera prise en compte et intégrée aux éléments de la base de données. L'on trouvera une synthèse récente des problèmes soulevés par la constitution d'une base de données prosopographiques en histoire, ainsi que quelques exemples de bases dans J. АкокA, I. CоміN-WATTTiAu et C. Du MouzA, "Conception de base de données prosopographiques en histoire : un état de l'art », Revue ouverte des systèmes d'information, 1/3 (2020), p. 1-19.

50. Voir J.-M. MOEGLIN, «Nation et nationalisme du Moyen Âge à l'époque moderne (FranceAllemagne) », Revue historique, 123/3 (1999), p. 537-554.

51. Selon la philosophie des Humanités numériques, cette nouvelle édition de Giovanni della Grossa doit favoriser le partage des connaissances et la collaboration entre chercheurs. Il sera notamment possible à des chercheurs extérieurs au projet initial de proposer des corrections, des notes complémentaires relatives à la Corse et au contexte méditerranéen, des informations sur des sites archéologiques ou des références de documents d'archives inédits à travers une plateforme d'échanges. Après validation par les coordinateurs du projet, ces éléments seront publiés sur la base sous la signature de leur propre auteur.

52. Voir V. MARCHI VAN CAUWELAERT, « Pour une histoire de la Méditerranée vue des îles. Approche comparée de la Corse, de la Sardaigne et de la Sicile au bas Moyen Âge ", in Histoire monde, jeux d'échelles et espaces connectés, Paris, 2017, p. 95-110.

53. Depuis le départ à la retraite de J.-A. Cancellieri en 2019, la discipline ne compte qu'un seul maître de conférences. Les financements obtenus pour la période 2018-2021 ont permis de recruter un post-doctorat pendant deux ans, de réaliser des missions archivistiques en Italie et en Espagne, de solliciter un prestataire externe pour les missions archéologiques en l'absence d'un archéologue médiéviste à l'université de Corse, de recourir aux services d'un prestataire en informatique. La recherche de nouvelles sources de financement pour la période 2022-2025 est en cours.

\section{INDEX}

Index géographique : Corse

Mots-clés : Moyen Âge, chroniques, archives, fortifications, Humanités numériques, Giovanni della Grossa

\section{AUTEURS}

VANNINA MARCHI VAN CAUWELAERT

UMR 6240 LISA 
JEAN-ANDRÉ CANCELLIERI

UMR 6240 LISA

LUCIE ARRIGHI

EA 4083 CLEA

MARC BONNANT

Cyrnoweb

ÉMILIE TOMAS

Arkemine

MICHĖLE FERRARA

UMR 6240 LISA 